\title{
Prognostic biomarkers in individuals with prevalent coronary heart disease
}

\author{
Sharif A. Halim and L. Kristin Newby* \\ From the Duke Clinical Research Institute, Duke University Medical Center, Durham, NC, USA
}

\begin{abstract}
Coronary disease is the leading killer of individuals worldwide and a leading cause of healthcare expenditure. On a global scale, ischemic heart disease kills over 6 million individuals each year and is projected by the World Health Organization to be the greatest single-disease cause of death worldwide by an increasing margin into 2030. Nearly 17 million Americans (7.6\% of the population) have prevalent coronary heart disease, 8 million of whom have had a prior myocardial infarction. It is estimated that in 2009, 550,000 will die from coronary heart disease in the United States and that the direct and indirect costs from treating coronary heart disease will exceed $\$ 165$ billion. Although patients with known coronary artery disease are among the highest risk patients for future cardiac events, not all patients with coronary disease will have an ischemic event (first or recurrent). Determining which of these patients will have an ischemic event is critical to the concept of personalized cardiovascular care. Increasingly, biomarkers that can be readily assayed from blood or other body fluids will be critical to risk stratification and effective application of secondary prevention strategies, just as they have played an increasingly prominent role in risk stratification of acute coronary syndrome patients.
\end{abstract}

Keywords: Coronary heart disease, prognosis, biomarkers

\section{Introduction}

Ischemic heart disease is the leading cause of death and disability in the United States and worldwide. As the population ages and the epidemic of obesity and diabetes ensues, the prevalence of ichemic heart disease and risk for acute coronary events worldwide is expected to increase dramatically in the coming years. Although deaths from all forms of cancer combined are projected by the World Health Organization to exceed those from ischemic heart disease, the leading individual cause of death globally in 2030 is projected to be ischemic heart disease. Currently, approximately 17 million Americans have coronary heart disease, 8 million of whom have had a prior myocardial infarction (MI), and coronary heart disease accounts for over 550,000 deaths each year -1 in 5 deaths among both

${ }^{*}$ Corresponding author: L. Kristin Newby, MD, MHS, Duke Clinical Research Institute, P.O. Box 17969, Durham, NC 27715-7969, USA. Tel:: +1 919668 8805; Fax: +1 919668 7056; E-mail: newby001@mc.duke.edu. men and women. It is estimated that in the United States in 2009, 1.4 million individuals will suffer an acute MI - 700,000, a first acute MI; 500,000, a recurrent MI and another nearly 200,000, a silent first MI [1]. Further, survivors of coronary heart disease events are at high risk for recurrent coronary events, other vascular events and downstream consequences of coronary events. Within six years after a documented MI, $18 \%$ of men and $35 \%$ of women have a re-MI, $7 \%$ of men and $6 \%$ of women experience sudden death, approximately $22 \%$ of men and $46 \%$ of women will be disabled with heart failure, and $8 \%$ of men and $11 \%$ of women will have a stroke [1]. The estimated direct and indirect costs of treating coronary heart disease in the United States in 2009 are projected to be $\$ 165$ billion.

Atherosclerosis and atherothrombosis, which underpin ischemic heart disease, are complex processes involving multiple pathophysiologic components (calcification, neoangiogenesis, intraplaque hemorrhage, and vascular repair and stabilization mediated by bonemarrow derived "atheroprotective" cells) intertwined in an inflammatory milieu of atherogenic lipids and white 
blood cell and vascular endothelial and smooth muscle cell response, which are necessary, but not sufficient to result in a clinical cardiac event. On this pathophysiological background, randomized clinical trials have provided abundant evidence for therapies that can substantially reduce the risk of MI or death, but despite therapy with 4-5 drugs that modulate key mediators of atherothrombosis, including lipids, thrombosis and inflammation, cardiac events continue to occur [2-6]. A better system for risk stratification for ischemic heart disease events should enable improved targeting of existing therapies to those at greatest risk and most likely to benefit, at the same time reducing potential side effects, adverse reactions and unnecessary costs among patients at lower risk for an event where the benefit-risk balance from treatment is less favorable.

The use of prognostic biomarkers derived from blood and other body fluids holds great promise to refine clinical risk stratification of individuals with prevalent coronary heart disease and, in conjunction, to strategically guide treatment to those most likely to benefit. In this review we will consider available and emerging prognostic biomarkers for patients with prevalent coronary heart disease.

\section{Acute coronary syndromes: What have we learned?}

In cardiovascular risk assessment, the greatest activity in the study of prognostic biomarkers in individuals with prevalent coronary artery disease has been in the area of acute coronary syndromes (ACS). Patients with ACS are not the focus of this review, but represent a special subset of patients with known coronary disease who are at high risk for recurrent events, particularly over the first 3-6 months after their acute presentation with unstable angina or myocardial infarction (non-ST-segment elevation or ST-segment elevation). Each year, hundreds of reports of relationships of various biomarkers with clinical outcomes after ACS can be found on PubMed and they have been summarized previously $[7,8]$. However, there remain only a handful of biomarkers with sufficient evidence to support recommendations for their routine use in risk stratification of ACS patients in clinical practice [9]. These assays include the troponins (I and T), high-sensitivity $\mathrm{C}$-reactive protein (hsCRP) and b-type natriuretic peptide (BNP) and N-terminal proBNP (NT-proBNP). As such, each of these biomarkers, alone and in combination, has demonstrated incremental contribution to risk stratification in the context of routinely available clinical and laboratory information such as vital signs, electrocardiographic findings and the serum creatinine (or estimated creatinine clearance). Importantly, there are high-quality commercially available assays for use on standard laboratory instruments or point-of-care devices that make these biomarkers ideal for widespread application in clinical practice.

Study of biomarkers for prognosis after ACS has also provided additional important insights that should be applicable to biomarker assessment of prognosis among patient with prevalent but stable coronary heart diseases. First, the ability to combine prognostic markers to create profiles of risk has also evolved in the arena of ACS. In this paradigm, biomarkers representing the activity of various pathways involved in ACS (e.g., myocardial necrosis, inflammation, markers of platelet and vascular activity and the neurohormonal axis) are combined to provide a more comprehensive and overarching assessment of risk [10-12]. In each case, the prognostic capability of the combination of markers was greater than any of the individual markers or readily available clinical characteristics alone.

An important observation from the use of protein biomarkers for risk stratification in ACS, which may have particular relevance to chronic coronary heart disease, was that the value of a prognostic marker varies according to the clinical event of interest and to the time horizon over which it is being assessed. In this context, in a substudy of the GUSTO-IV trial, Westerhout and colleagues observed that while troponin T, NT-proBNP and hsCRP were each independently associated with 1-year mortality, only troponin $\mathrm{T}$ was independently associated with risk of subsequent MI [13]. In addition, they found that the time horizon over which risk was assessed was important in determining the utility of a prognostic marker. In this case, while both NTproBNP and troponin T were associated with both 30day and 1-year mortality, hsCRP was not significantly associated with 30-day death. Additionally, the prognostic utility of troponin $\mathrm{T}$ for the composite of death or MI was strongest in the short term, whereas that for NT-proBNP was diminished at 30-days compared with 1-year.

Finally, perhaps the most important insights to validation of biomarkers for use in clinical practice was finding that the result from testing a prognostic biomarker identified patients who received incremental benefit from various treatments; thus, the biomarker could be used to guide therapeutic decision-making to reduce a patient's risk of ischemic events. In the case of 
ACS, the best evidence for use of a biomarker to guide treatment is for the troponins. Several studies have now demonstrated that patients who are troponin positive derive enhanced benefit from the use of glycoprotein IIb/IIIa inhibitors, low-molecular-weight heparins and early coronary angiography [14-17], such that use can be individually directed by the results of biomarker testing to those who will most benefit from therapy, avoiding potential risks of therapy in those unlikely to benefit.

\section{Prognostic biomarkers in patients with prevalent, stable coronary heart disease}

\subsection{Inflammation and risk}

Although fewer studies have assessed biomarkers for risk stratification and therapeutic decision-making in patients with prevalent but stable coronary heart disease, several important observations have been made and new prognostic biomarkers are emerging. However, as with ACS, only a handful of biomarkers are both of independent prognostic value and readily available for clinical testing. Only hsCRP has been associated with therapeutic intervention to mitigate the heightened risk associated with biomarker elevation.

The view of coronary artery disease as an inflammatory process is now widely accepted [18]. It is also well known that treatments, such as statins, that reduce the risk of coronary events also reduce evidence of inflammation. One of the most striking examples of this interrelationship is demonstrated by hsCRP. Not only is hsCRP an independent biomarker of risk for cardiovascular events, reductions in clinical events by treatment with statins are associated with reductions in hsCRP, and patients with higher hsCRP levels derive the greatest benefit from statin treatment. In the Cholesterol and Recurrent Events (CARE) trial, in which patients with known coronary artery disease and elevated cholesterol levels were randomized to pravastatin or placebo, patients with higher hsCRP levels were not only at greater risk for subsequent death or MI, they were also more likely to benefit from treatment with pravastatin, which was also associated with a greater decrease in hsCRP levels [19,20]. Similar findings have been associated with other statin agents when examined in both primary and secondary prevention [21-24], and in studies of atherosclerosis regression. In the REVERSAL study, intensive statin treatment reduced the rate of progression of atherosclerosis detected by intravascular ultra- sound, a finding that was correlated with reductions in hsCRP [25]. Most recently, in the primary prevention setting, in the JUPITER trial patients with elevated hsCRP but cholesterol levels below current guidelines recommendations for treatment derived benefit from treatment with rosuvastatin compared with placebo, suggesting that hsCRP as a biomarker of inflammation is not only a prognostic indicator but may be used to guide therapy [26]. How or if this finding in primary prevention will translate to modulation of statin therapy in secondary prevention is as yet unknown.

Other markers of inflammation have also been associated with both progression of and prognosis in chronic coronary disease and provide incremental information to that from hsCRP. In a study of coronary disease progression in patients awaiting angioplasty for a mean of 4.8 months, rapid progression of known coronary disease was associated not only with higher levels of hsCRP but also with other markers of the inflammatory process including neopterin $(P<0.001)$, matrix metalloproteinase-9 $(P=0.002)$, and soluble intercellular adhesion molecule $1(P<0.001)$ [27]. Further, in a biomarker substudy of the $\mathrm{Z}$ phase of $\mathrm{A}$ to $\mathrm{Z}$ trial of early intensive vs. delayed modest-dose simvastatin treatment for long-term secondary prevention after an ACS, de Lemos and colleagues demonstrated that monocyte chemoattractant protein-1 (MCP-1) added incremental prognostic information to clinical characteristics and hsCRP in both short- and long-term risk stratification for mortality (HR 2.16 [95\% confidence interval 1.54-3.02] [28]. However, there was not incremental treatment benefit from intensive statin therapy in patients with elevated MCP-1.

\subsection{NT-proBNP and risk in chronic coronary heart disease}

As with hsCRP, NT-proBNP, a marker of neurohormonal status, has been shown to be a marker of risk not only in ACS but also in the larger population of patients with chronic stable coronary heart disease. In a study of 1059 patients with chronic stable angina and angiographically confirmed obstructive coronary disease followed for a mean of 3.6 years, NT-proBNP levels were strongly associated with mortality (HR 5.83 [95\% confidence interval: $2.07-16.44$ ] for the fourth versus the first quartile) and cardiovascular mortality (HR, 5.98 [1.55-23.13] for the fourth versus the first quartile) after adjustment for New York Heart Association (NYHA) class and other clinical covariates [29]. Among patients with NYHA I or II, the association of 
NT-proBNP with mortality was particularly strong: HR 6.03 [2.07-17.52] for the fourth vs. the first quartile. A second study of NT-pro-BNP in 1034 patients referred for angiography for evaluation of suspected coronary heart disease also revealed a significant independent association of NT-proBNP levels with mortality during long-term follow up [30]. In this case, the adjusted HR for mortality comparing the fourth vs. first quartile was 2.4 [1.5-4.0]. Importantly, among other clinical covariates, the model adjusted for age, history of MI or chronic heart failure, ejection fraction and extent of coronary disease.

Thus, NT-proBNP appears to be an important prognostic marker among patients with chronic coronary heart disease. However, unlike hsCRP, it remains to be elucidated what, if any, therapeutic interventions may be specifically undertaken to reduce the risk associated with elevated levels of NT-proBNP.

\subsection{New markers of risk and treatment benefit}

\subsubsection{Growth differentiation factor-15 (GDF-15)}

GDF-15, a member of the TGF-B superfamily, is produced by activated macrophages within atherosclerotic lesions and may be a maker of plaque activity and risk for subsequent coronary events. To date there are no data specifically from chronic coronary disease populations, but GDF-15 was independently associated with death, MI or stroke in primary prevention in the Women's Health Study (odds ratio 2.7 [1.6-4.9]) [31]. In addition, in the setting of both ST-segment elevation (STE) MI and non-ST-segment elevation (NSTE) ACS GDF-15 has been independently associated with risk for subsequent cardiovascular events. GDF-15 was independently associated with mortality in 741 STEMI patients enrolled in the Assessment of the Safety and Efficacy of a New Thrombolytic (ASSENT)-2 and ASSENT-plus trials [32]. Similarly, in 2081 patients with NSTE ACS enrolled in the GUSTO IV trial, 1year mortality increased from $1.5 \%$ to $5.0 \%$ to $14.1 \%$ with increasing tertiles of GDF-15 [33]. In a multivariable Cox regression model, GDF-15 along with NTproBNP were the only biomarkers independently associated with mortality risk. In a second NSTE ACS dataset from the Fast Revascularization during InStability in Coronary artery disease II (FRISC-II) trial, not only was GDF-15 associated with increased risk of death or MI over a median follow up of 2 years, there was also a significant interaction with treatment such that patients with the highest levels of GDF-15 derived the greatest benefit from an early invasive strat- egy [34]. If these findings translate to chronic coronary disease populations, GDF-15 may be a powerful tool for both risk assessment and to identify patients for specific treatment modifications to mitigate risk.

\subsubsection{Cystatin $C$}

Renal disease is an important factor in the development of cardiovascular disease, and in patients with chronic coronary disease or at high risk for coronary disease has been shown to be associated with cardiovascular events $[35,36]$. Further, in the HOPE trial, not only was renal insufficiency a predictor of outcomes, it also identified a population that preferentially benefited from ramipril treatment [36]. Cystatin C is a cysteine protease inhibitor that is constitutively secreted by most nucleated cells [37], and has been shown to be a more consistent measure of renal function than creatinine or estimated glomerular filtration rate [38]. Cystatin $\mathrm{C}$ has been shown to be predictive of clinical outcomes in community-dwelling elderly patients without known coronary disease and among patients with NSTE ACS [39,40].

In 1033 patients with coronary heart disease, Koenig and colleagues demonstrated that of creatinine, estimated creatinine clearance and cystatin $\mathrm{C}$, only cystatin $\mathrm{C}$ was significantly associated with secondary cardiovascular events in follow-up. After adjustment for clinical covariates, the HR for the 5th quintile compared with the 1st quintile was 2.27 [1.05-4.91] [41]. Similarly, Shlipak and colleagues demonstrated the independent contribution of cystatin $\mathrm{C}$ to risk assessment in 979 subjects with documented coronary artery disease who were enrolled in the Heart and Soul Study. Over 3.5 years of follow-up, 142 individuals suffered the composite endpoint of death, MI, or stroke. Cystatin $\mathrm{C}$ was associated with the composite endpoint with a HR of 1.72 (95\% CI, 1.10-2.70) after adjustment for clinical covariates [42]. However, after consideration of other biomarkers of risk that were also independent of clinical characteristics (hsCRP, NT-proBNP, interleukin-6 and albuminuria), only NTproBNP, hsCRP and albuminuria remained independently associated with outcome. These findings were similar to those of Luc and colleagues in 9758 men aged 50-59 in the primary prevention Prospective Epidemiological Study of Myocardial Infarction (PRIME) Study in which cystatin $\mathrm{C}$ was independent of clinical predictors of cardiovascular events, but was not statistically significant after adjustment for hsCRP [43]. These results support that a measure of renal function is a critical component of risk stratification, but that 
perhaps a readily available, inexpensive marker of renal impairment such as albuminuria and other available biomarkers of risk may be satisfactory in risk prognostication in stable patients with coronary heart disease.

\subsubsection{Lp-PLA2}

Perhaps the most intriguing new biomarker of prognosis for patients with coronary heart disease is lipoprotein-associated phospholipase A2 (Lp-PLA2). Lp-PLA2 produced in activated macrophages generates proinflammatory and proatherogenic products through the hydrolysis of phospholipids. Both Lp-PLA2 activity and mass and activity assays have been independently associated with coronary heart disease events in numerous studies of both primary and secondary prevention populations $[44,45]$. In fact, the emerging evidence for Lp-PLA2 as a biomarker of risk was so compelling that a consensus panel was convened to review the available data and update recommendations for the for use of Lp-PLA2 in clinical practice [46]. The consensus panel proposed the use of Lp-PLA2 as an adjunct for risk assessment in patients at moderate or high coronary disease risk according to the Framingham risk classification as changes in risk classification at these levels would lead to institution of or intensification of lipid lowering therapy and lowering of the target LDL goal; however, it stopped short of recommending treatment specifically for elevated Lp-PLA2.

Several studies now suggest that treatment specifically targeted at Lp-PLA2 may be of clinical benefit. In a swine model, inhibition of Lp-PLA2 activity by a specific inhibitor of Lp-PLA2, darapladib, reduced the development of complex atherosclerotic plaques [47]. However, in the Dallas Heart study the level of LpPLA2 activity or mass measurement was not associated with measures of atherosclerosis (coronary artery calcium, abdominal aortic plaque or aortic wall thickness) in women and only Lp-PLA2 mass was independently associated with coronary artery calcium in men [48]. The authors postulated that the association of Lp-PLA2 with risk may be mediated more by modulation of plaque instability than by plaque development or progression. This postulation is supported by the results of a randomized clinical trial of 12 months of darapladib treatment vs. placebo on a primary endpoint of coronary atheroma deformability and hsCRP with a secondary endpoint of necrotic core size and atheroma size in 330 patients with documented coronary artery disease [49]. After 12 months, there was no significant difference between treatment groups on either of the primary endpoints. However, darapladib stopped necrotic core expansion compared with continued increase in the placebo group, suggesting a potential mechanism for modulating plaque vulnerability. In a second randomized trial with intermediate endpoints conducted in 959 patients on a background of atorvastatin therapy in all patients, darapladib treatment resulted in both a sustained inhibition of Lp-PLA2 activity and decreases in both IL-6 (12\%) and hsCRP (13\%) compared with placebo with no adverse effects [50].

Based on these preliminary studies, darapladib is now undergoing phase III testing in the randomized, placebo controlled STABILITY trial of prevention of cardiovascular clinical events in patients with known coronary artery disease who are already treated with statin therapy.

\section{Summary}

Millions of individuals worldwide are affected by coronary artery disease and are at risk for death or initial or recurrent myocardial infarction and its downstream complications. A number of biomarkers are currently available in clinical practice to assess prognosis in these patients (hsCRP, NT-proBNP, markers of renal function and the newly emerged Lp-PLA2), and novel biomarkers continue to emerge. In the case of hsCRP, treatment with statins has enhanced benefit among patients with elevated hsCRP and may be used to guide therapy. New recommendations are available for the use of Lp-PLA2 as a biomarker of risk that has been associated independently of hsCRP with ischemic heart disease events. Whether treatment to inhibit LpPLA2 will reduce cardiovascular events, elevating it to the status of a risk factor, remains to be proved in an ongoing randomized clinical trial, but could be the next major breakthrough in treatment of chronic coronary heart disease.

\section{References}

[1] D. Lloyd-Jones, R. Adams, M. Carnethon et al., Heart disease and stroke statistics 2009 update: a report from the American Heart Association Statistics Committee and Stroke Statistics Subcommittee, Circulation 119 (2009), e21-e181.

[2] The Long-Term Intervention with Pravastatin in Ischaemic Disease (LIPID) Study Group, Prevention of cardiovascular events and death with pravastatin in patients with coronary heart disease and a broad range of initial cholesterol levels, $N$ Engl J Med 339 (1998), 1349-1357.

[3] Heart Outcomes Prevention Evaluation Study Investigators, Effects of an angiotensin-converting-enzyme inhibitor, ramipril, on cardiovascular events in high-risk patients, $N$ Engl J Med 342 (2000), 145-153. 
[4] Antithrombotic Trialists Collaboration, Collaborative metaanalysis of randomised trials of antiplatelet therapy for prevention of death, myocardial infarction, and stroke in high risk patients, BMJ 324 (2002), 71-86.

[5] CAPRIE Steering Committee, A randomised, blinded, trial of clopidogrel versus aspirin in patients at risk of ischaemic events (CAPRIE), Lancet 348 (1996), 1329-1339.

[6] The Clopidogrel in Unstable Angina to Prevent Recurrent Events Trial Investigators, Effects of clopidogrel in addition to aspirin in patients with acute coronary syndromes without ST-segment elevation, N Engl J Med 345 (2001), 494-502.

[7] F.S. Apple, A.H. Wu, J. Mair, J. Ravkilde, M. Panteghini, J. Tate, F. Pagani, R.H. Christenson, M. Mockel, O. Danne et al., for the Committee on Standardization of Markers of Cardiac Damage of the IFCC, Future biomarkers for detection of ischemia and risk stratification in acute coronary syndrome, Clin Chem 51 (2005), 810-824.

[8] G.S. Ginsburg, M. Donahue and L.K. Newby, Prospects for personalized cardiovascular medicine: the impact of genomics, J Am Coll Cardiol 46 (2005), 1615-1627.

[9] D.A. Morrow, C.P.Cannon, R.L. Jesse, L.K. Newby, J. Ravkilde, A.B. Storrow, A.H.B. Wu and R.H. Christenson, National Academy of Clinical Biochemistry Laboratory Medicine Practice Guidelines: clinical characteristics and utilization of biochemical markers in acute coronary syndromes, Circulation 115 (2007), e356-375.

[10] C. Heeschen, S. Dimmeler, C.W. Hamm, S. Fichtlscherer, M.L. Simoons and A.M. Zeiher, for the CAPTURE Investigators. Pregnancy-associated plasma protein-A levels in patients with acute coronary syndromes: comparison with markers of systemic inflammation, platelet activation, and myocardial necrosis, J Am Coll Cardiol 45 (2005), 229-237.

[11] M.S. Sabatine, D.A. Morrow, J.A. de Lemos, C.M. Gibson, S.A. Murphy, N. Rifai, C. McCabe, E.M. Antman, C.P. Cannon and E. Braunwald, Multimarker approach to risk stratification in non-ST elevation acute coronary syndromes: simultaneous assessment of troponin I, C-reactive protein, and B-type natriuretic peptide, Circulation 105 (2002), 1760-1763.

[12] S. Baldus et al., Myeloperoxidase serum levels predict risk in patients with acute coronary syndromes, Circulation $\mathbf{1 0 8}$ (2003), 1440-1445.

[13] C.M. Westerhout, Y. Fu, M.S. Lauer, S. James, P.W. Armstrong, E. Al-Hattab, R.M. Califf, M.L. Simoons, L. Wallentin and E. Boersma, for the GUSTO-IV ACS Trial Investigators, Short- and long-term risk stratification in acute coronary syndromes: the added value of quantitative ST-segment depression and multiple biomarkers, J Am Coll Cardiol 48 (2006), 939-947.

[14] D.A. Morrow, E.M. Antman, M. Tanasijevic, N. Rifai, J.A. de Lemos, C.H. McCabe, C.P. Cannon and E. Braunwald, Cardiac troponin I for stratification of early outcomes and the efficacy of enoxaparin in unstable angina: a TIMI-11B substudy, J Am Coll Cardiol 36 (2000), 1812-1817.

[15] D.A. Morrow, C.P. Cannon, N. Rifai, M.J. Frey, R. Vicari, N. Lakkis, D.H. Robertson, D.A. Hille, P.T. DeLucca, P.M. DiBattiste et al., Ability of minor elevations of troponins I and $\mathrm{T}$ to predict benefit from an early invasive strategy in patients with unstable angina and non-ST elevation myocardial infarction: results from a randomized trial, JAMA 286 (2001), 2405-2412.

[16] L.K. Newby, E.M. Ohman, R.H. Christenson, D.J. Moliterno, R.A. Harrington, H.D. White, P.W. Armstrong, F. Van de Werf, M. Pfisterer, V. Hasselblad et al., Benefit of glycoprotein IIb/IIIa inhibition in patients with acute coronary syn- dromes and troponin t-positive status: the paragon-B troponin T substudy, Circulation 103 (2001), 2891-2896.

[17] C. Heeschen, C.W. Hamm, B. Goldmann, A. Deu, L. Langebrink and H.D. White, for the PRISM Study Investigators. Troponin concentrations for stratification of patients with acute coronary syndromes in relation to therapeutic efficacy of tirofiban, Lancet 354 (1999), 1757-1762.

[18] R. Ross, Atherosclerosis: an inflammatory disease, $N$ Engl J Med 340 (1999), 115-126.

[19] P.M. Ridker, N. Rifai, M.A. Pfeffer, F.M. Sacks, L.A. Moye, S. Goldman, G.C. Flaker and E. Braunwald, for the Cholesterol and Recurrent Events (CARE) investigators. Inflammation, pravastatin, and the risk of coronary events after myocardial infarction in patients with average cholesterol levels, Circulation 98 (1998), 839-844.

[20] P.M. Ridker, N. Rifai, M.A. Pfeffer, F. Sacks and E. Braunwald, for the Cholesterol and Recurrent Events (CARE) Investigators. Long-term effects of pravastatin on plasma concentration of C-reactive protein, Circulation 100 (1999), 230 235.

[21] P.M. Ridker, N. Rifai, M. Clearfield, J.R. Downs, S.E. Weis, J.S. Miles and A.M. Gotto, for the Air Force/Texas Coronary Atherosclerosis Prevention Study Investigators. Measurement of C-reactive protein for the targeting of statin therapy in the primary prevention of acute coronary events, $N$ Engl J Med 344 (2001), 1959-1965.

[22] M.A. Albert, E. Danielson, N. Rifai and P.M. Ridker, Effect of statin therapy of C-reactive protein levels: the pravastatin inflammation/CRP evaluation (PRINCE): a randomized trial and cohort study, JAMA 286 (2001), 64-70.

[23] P.M. Ridker, N. Rifai and S.P. Lowenthal, Rapid reduction in C-reactive protein with cerivastatin among 785 patients with primary hypercholesterolemia, Circulation 103 (2001), 11911193.

[24] I. Jialal, C. Stein, D. Balis, S.M. Grundy, B. Adams-Huet and S. Deveraj, Effect of hydroxymethyl glutaryl coenzyme A reductase inhibitor therapy on high sensitive C-reactive protein levels, Circulation 103 (2001), 1933-1935.

[25] S.E. Nissen, E.M. Tuzcu, P. Schoenhagen, B.G. Brown, P. Ganz, R.A. Vogel, T. Crowe, G. Howard, C.J. Cooper, B. Brodie, C.L. Grines, A.N. DeMaria, REVERSAL Investigators, Effect of intensive compared with moderate lipidlowering therapy on progression of coronary atherosclerosis: a randomized controlled trial, JAMA 291 (2004), 1071-1080.

[26] P.M. Ridker, E. Danielson, F.A. Fonseca, J. Genest, A.M. Gotto Jr., J.J. Kastelein, W. Koenig, P. Libby, A.J. Lorenzatti, J.G. MacFadyen, B.G. Nordestgaard, J. Shepherd, J.T. Willerson and R.J. Glynn, JUPITER Study Group. Rosuvastatin to prevent vascular events in men and women with elevated Creactive protein, N Engl J Med 359 (2008), 2195-2207.

[27] E. Zouridakis, P. Avanzas, R. Arroyo-Espliguero, S. Fredericks and J.C. Kaski, Markers of inflammation and rapid coronary artery disease progression in patients with stable angina pectoris, Circulation 110 (2004), 1747-1753.

[28] J.A. de Lemos, D.A. Morrow, M.A. Blazing, P. Jarolim, S.D. Wiviott, M.S. Sabatine, R.M. Califf and E. Braunwald, Serial measurement of monocyte chemoattractant protein-1 after acute coronary syndromes: results from the A to $\mathrm{Z}$ trial, $J \mathrm{Am}$ Coll Cardiol 50 (2007), 2117-2124.

[29] G. Ndrepepa, S. Braun, K. Niemöller, J. Mehilli, N. von Beckerath, O. von Beckerath, W. Vogt, A. Schömig and A. Kastrati, Prognostic value of N-terminal pro-brain natriuretic peptide in patients with chronic stable angina, Circulation 112 (2005), 2102-2107. 
[30] C. Kragelund, B. Grønning, L. Køber, P. Hildebrandt and R. Steffensen, N-terminal pro-B-type natriuretic peptide and long-term mortality in stable coronary heart disease, $N$ Engl $J$ Med 352 (2005), 666-675.

[31] D.A. Brown, S.N. Breit, J. Buring, W.D. Fairlie, A.R. Bauskin, T. Liu and P.M. Ridker, Concentration in plasma of macrophage inhibitory cytokine-1 and risk of cardiovascular events in women: a nested case-control study, Lancet 359 (2002), 2159-2163.

[32] T. Kempf, E. Björklund, S. Olofsson, B. Lindahl, T. Allhoff, T. Peter, J. Tongers, K.C. Wollert and L. Wallentin, Growth-differentiation factor-15 improves risk stratification in ST-segment elevation myocardial infarction, Eur Heart $\mathbf{J} \mathbf{2 8}$ (2007), 2858-2865.

[33] K.C. Wollert, T. Kempf, T. Peter, S. Olofsson, S. James, N. Johnston, B. Lindahl, R. Horn-Wichmann, G. Brabant, M.L. Simoons, P.W. Armstrong, R.M. Califf, H. Drexler and L. Wallentin, Prognostic value of growth-differentiation factor15 in patients with non-ST-elevation acute coronary syndrome, Circulation 115 (2007), 962-971.

[34] K.C. Wollert, T. Kempf, B. Lagerqvist, B. Lindahl, S. Olofsson, T. Allhoff, T. Peter, A. Siegbahn, P. Venge, H. Drexler and L. Wallentin, Growth differentiation factor 15 for risk stratification and selection of an invasive treatment strategy in non ST-elevation acute coronary syndrome, Circulation 116 (2007), 1540-1548.

[35] M.J. Sarnak, A.S. Levey, A.C. Schoolwerth, J. Coresh, B. Culleton, L.L. Hamm et al., Kidney disease as a risk factor for development of cardiovascular disease, Circulation 108 (2003), 2154-2169.

[36] J.F. Mann, H.C. Gerstein, J. Pogue, J. Bosch and S. Yusuf, Renal insufficiency as a predictor of cardiovascular outcomes and the impact of ramipril: the HOPE randomized trial, Ann Intern Med 134 (2001), 629-636.

[37] M. Abrahamson, A. Grubb, I. Olafsson and A. Lundwall, Molecular cloning and sequence analysis of cDNA coding for the precursor of the human cysteine proteinase inhibitor cystatin C, FEBS Lett 216 (1987), 229-233.

[38] S. Herget-Rosenthal, S. Trabold, F. Pietruck, M. Holtmann, T. Philipp and A. Kribben, Cystatin C: efficacy as screening test for reduced glomerular filtration rate, Am J Nephrol $\mathbf{2 0}$ (2000), 97-102.

[39] M.G. Shlipak, M.J. Sarnak, R. Katz, L.F. Fried, S.L. Seliger, A.B. Newman, D.S. Siscovick and C. Stehman-Breen, Cystatin $\mathrm{C}$ and the risk of death and cardiovascular events among elderly persons, $N$ Engl J Med 352 (2005), 2049-2060.

[40] T. Jernberg, B. Lindahl, S. James, A. Larsson, L.O. Hansson and L. Wallentin, Cystatin C: a novel predictor of outcome in suspected or confirmed non-ST-elevation acute coronary syndrome, Circulation 110 (2004), 2342-2348.

[41] W. Koenig, D. Twardella, H. Brenner and D. Rothenbacher, Plasma concentrations of cystatin $\mathrm{C}$ in patients with coronary heart disease and risk for secondary cardiovascular events: more than simply a marker of glomerular filtration rate, Clin Chem 51 (2005), 321-327.

[42] M.G. Shlipak, J.H. Ix, K. Bibbins-Domingo, F. Lin and M.A. Whooley, Biomarkers to predict recurrent cardiovascular disease: the Heart and Soul Study, Am J Med 121 (2008), 50-57.

[43] G. Luc, J.M. Bard, C. Lesueur, D. Arveiler, A. Evans, P. Amouyel, J. Ferrieres, I. Juhan-Vague, J.C. Fruchart and P. Ducimetiere, PRIME Study Group. Plasma cystatin-C and development of coronary heart disease: The PRIME Study, Atherosclerosis 185 (2006), 375-380.

[44] J.L. Anderson, Lipoprotein-associated phospholipase A2: an independent predictor of coronary artery disease events in primary and secondary prevention, Am J Cardiol 101 (2008), 23F-33F.

[45] M.A. Corson, P.H. Jones and M.H. Davidson, Review of the evidence for the clinical utility of lipoprotein-associated phospholipase A2 as a cardiovascular risk marker, Am J Cardiol 101 (2008), 41F-50F.

[46] M.H. Davidson, M.A. Corson, M.J. Alberts, J.L. Anderson, P.B. Gorelick, P.H. Jones, A. Lerman, J.P. McConnell and H.S. Weintraub, Consensus panel recommendation for incorporating lipoprotein-associated phospholipase A2 testing into cardiovascular disease risk assessment guidelines, Am J Cardiol 101 (2008), 51F-57F.

[47] R.L. Wilensky, Y. Shi, E.R. Mohler 3rd, D. Hamamdzic, M.E. Burgert, J. Li, A. Postle, R.S. Fenning, J.G. Bollinger, B.E. Hoffman, D.J. Pelchovitz, J. Yang, R.C. Mirabile, C.L. Webb, L. Zhang, P. Zhang, M.H. Gelb, M.C. Walker, A. Zalewski and C.H. Macphee, Inhibition of lipoprotein-associated phospholipase A2 reduces complex coronary atherosclerotic plaque development, Nat Med 14 (2008), 1059-1066.

[48] E.S. Brilakis, A. Khera, B. Saeed, S. Banerjee, D.K. McGuire, S.A. Murphy and J.A. de Lemos, Association mass and activity with coronary and aortic atherosclerosis: findings from the Dallas Heart Study, Clin Chem 54 (2008), 1975-1981.

[49] P.W. Serruys, H.M. García-García, P. Buszman, P. Erne, S. Verheye, M. Aschermann, H. Duckers, O. Bleie, D. Dudek, H.E. Bøtker, C. von Birgelen, D. D’Amico, T. Hutchinson, A. Zambanini, F. Mastik, G.A. van Es, A.F. van der Steen, D.G. Vince, P. Ganz, C.W. Hamm, W. Wijns and A. Zalewski, Integrated Biomarker and Imaging Study-2 Investigators. Effects of the direct lipoprotein-associated phospholipase A(2) inhibitor darapladib on human coronary atherosclerotic plaque, Circulation 118 (2008), 1172-1182.

[50] E.R. Mohler 3rd, C.M. Ballantyne, H.M. Davidson, M. Hanefeld, L.M. Ruilope, J.L. Johnson and A. Zalewski, Darapladib Investigators, The effect of darapladib on plasma lipoprotein-associated phospholipase A2 activity and cardiovascular biomarkers in patients with stable coronary heart disease or coronary heart disease risk equivalent: the results of a multicenter, randomized, double-blind, placebo-controlled study, J Am Coll Cardiol 51 (2008), 1632-1641. 




The Scientific World Journal


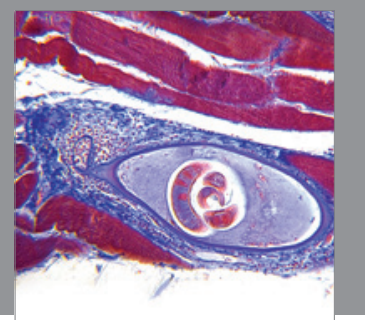

Gastroenterology

Research and Practice
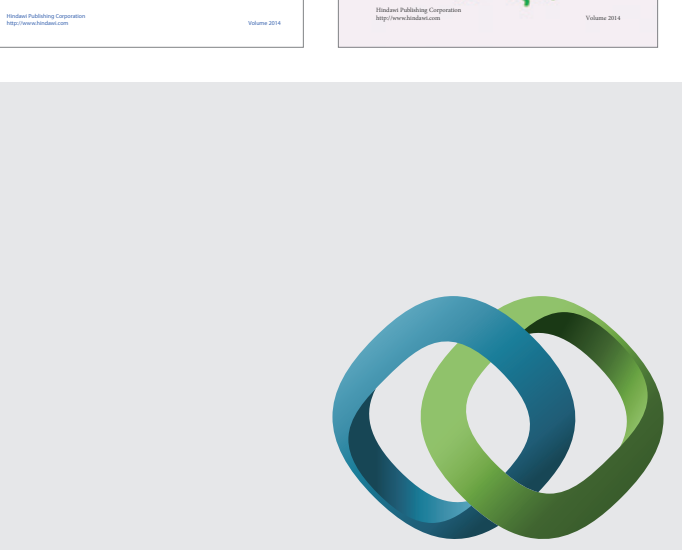

\section{Hindawi}

Submit your manuscripts at

http://www.hindawi.com


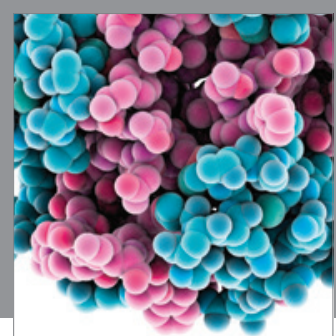

Journal of
Diabetes Research

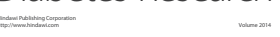

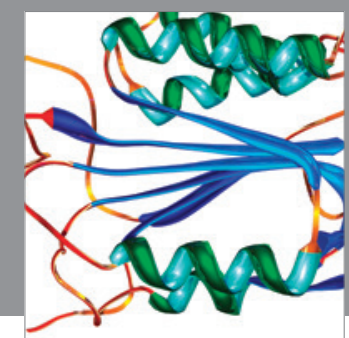

Disease Markers
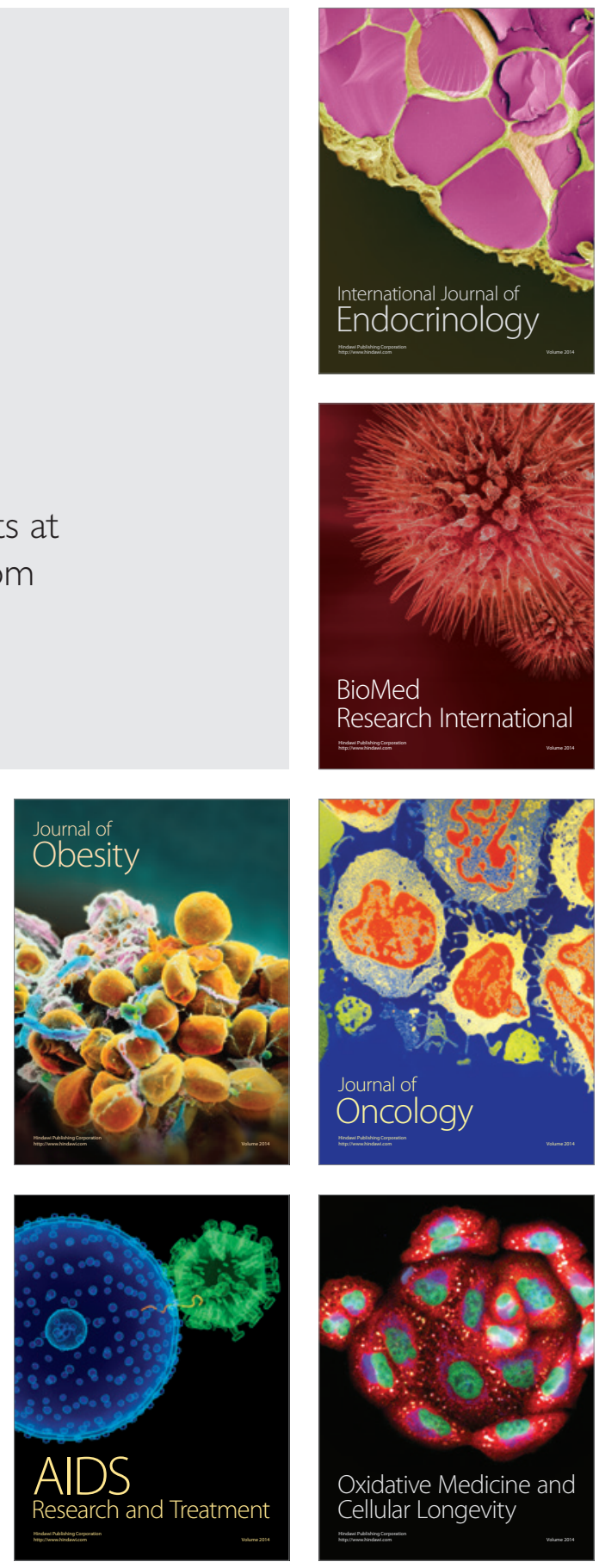